\title{
Omental Free-Shaped Flap Reinforcement on the Anastomosis and Dissected Area (OFFROAD) Following Reconstruction after Gastrectomy: A Retrospective Case-Control Study
}

\author{
Chul Kyu Roh, M.D., Sang-Uk Han, M.D., Ph.D. \\ Department of Surgery, Ajou University School of Medicine, Suwon, Korea
}

\begin{abstract}
The recent advancements in surgical techniques and perioperative care have improved postoperative morbidity and survival after gastric cancer surgeries. However, anastomotic leakage, the most serious complication post-gastrectomy, continues to occur. In esophageal and colorectal cancer surgeries, the omentum is used as a physical barrier and increases blood perfusion to prevent leakage to the anastomotic site. However, the use of the omentum as an anastomotic barrier after gastrectomy has not been reported yet. The authors aimed to evaluate the efficacy and safety of omental free-shaped flap reinforcement on the anastomosis and dissected area following reconstruction after gastrectomy for preventing and treating anastomotic leakage. They reported that omental free-shaped flap reinforcement on the anastomosis might prevent anastomotic leakage post-gastrectomy. The omental flap also prevented further deterioration when leakage occurred. However, anastomosis-related complications, such as anastomotic stenosis and delayed gastric emptying, after an omental patch technique need to be evaluated further.
\end{abstract}

Keywords: Gastric cancer, Surgery, Anastomosis, Omental flap, Omentopexy
Received February 3, 2020

Accepted February 25, 2020

Corresponding author

Sang-Uk Han

Department of Surgery, Ajou

University School of Medicine, 164

Worldcup-ro, Yeongtong-gu, Suwon 16499 Korea

Tel: +82-31-219-5200

Fax: +82-31-219-7860

E-mail: hansu@ajou.ac.kr ORCID:

https://orcid.org/0000-0001-5615-4162

This is an Open Access article distributed under the terms of the Creative Commons Attribution Non-Commercial License (http:// creativecommons.org/licenses/by-nc/4.0/) which permits unrestricted non-commercial use, distribution, and reproduction in any medium, provided the original work is properly cited.

Copyright $\odot 2020$ The Journal of Minimally Invasive Surgery. All rights reserved.
Remarkable technical advances in surgical procedures and systematically perioperative management have improved complication rates, quality of life, and even survival after gastric cancer surgery. However, some patients still suffer from major postoperative complications such as anastomotic leakage, which is the most serious complication after gastrectomy for gastric cancer., ${ }^{1,2}$ The anastomosis leakages delay recovery post-surgery, which may delay the initiation of adjuvant chemotherapy in patients who require it. Thus, major complications such as anastomotic leakage negatively affect survival., ${ }^{3,4}$ There have been many endeavors to prevent anastomotic leakage. In an effort to decrease anastomosis-related complications, many surgeons evaluated the risk factors for anastomotic leakage and changed anastomosis techniques and materials. In addition, biomedical barriers to anastomotic sites have been used for safe anastomosis. However, significant improvements have not yet been made in preventing anastomotic leakage.

The omentum as a biological barrier had been used to prevent leakage in pancreatic, esophageal, and colorectal cancer surgeries. Recent studies have reported favorable results of omental flaps in reducing leakage rates. ${ }^{5,6}$ At an anastomotic site, the omentum acts as a physical barrier and increases blood perfusion to the site. However, there have been no reports on the use of the omentum as a barrier to anastomosis after gastrectomy.

This case-control study aimed to evaluate efficacy and safety of omental free-shaped flap reinforcement on the 
anastomosis and dissected area following reconstruction after gastrectomy for preventing and treating anastomotic leakage. This is the first study to investigate the efficacy of anastomotic reinforcement using the omentum in gastric cancer surgery. There was no significant difference in the anastomotic leak rate. When anastomotic leakage occurred, leakage-related signs and symptoms were less observed in the omental patch group. The omentum could prevent peritoneal dissemination of intestinal fluid and septic complications after leakage. However, anastomosis-related complications such as delayed gastric emptying, stenosis, and leakage tended to be more observed in the omental patch group. Therefore, further studies are warranted to evaluate the usefulness of the omental flap for anastomotic reinforcement in gastric cancer surgeries in the future.

\section{ORCID}

Chul Kyu Roh, https://orcid.org/0000-0003-2254-6146

Sang-Uk Han, https://orcid.org/0000-0001-5615-4162

\section{AUTHORS' CONTRIBUTIONS}

Conceptualization: Sang-Uk Han. Formal analysis: Chul Kyu Roh. Methodology: Chul Kyu Roh. Writing-original draft: Chul Kyu Roh. Writing-review and editing: Sang-Uk Han.

\section{CONFLICT OF INTEREST}

None.

\section{FUNDING}

None.

\section{ACKNOWLEDGMENTS}

None.

\section{REFERENCES}

1) Degiuli M, Sasako M, Ponti A, et al. Randomized clinical trial comparing survival after D1 or D2 gastrectomy for gastric cancer. Br J Surg 2014;101:23-31.

2) Lee H-J, Hyung WJ, Yang H-K, et al. Short-term Outcomes of a Multicenter Randomized Controlled Trial Comparing Laparoscopic Distal Gastrectomy With D2 Lymphadenectomy to Open Distal Gastrectomy for Locally Advanced Gastric Cancer (KLASS-02RCT). Ann Surg 2019;270:983-991.

3) Sierzega M, Kolodziejczyk P, Kulig J, Polish Gastric Cancer Study G. Impact of anastomotic leakage on long-term survival after total gastrectomy for carcinoma of the stomach. Br J Surg 2010;97: 1035-1042.

4) Yoo HM, Lee HH, Shim JH, Jeon HM, Park CH, Song KY. Negative impact of leakage on survival of patients undergoing curative resection for advanced gastric cancer. J Surg Oncol 2011;104:734740.

5) Merad F, Hay JM, Fingerhut A, Flamant Y, Molkhou JM, Laborde Y. Omentoplasty in the prevention of anastomotic leakage after colonic or rectal resection: a prospective randomized study in 712 patients. French Associations for Surgical Research. Ann Surg 1998;227:179-186.

6) Chen L, Liu F, Wang K, Zou W. Omentoplasty in the prevention of anastomotic leakage after oesophagectomy: a meta-analysis. Eur J Surg Oncol 2014;40:1635-1640.

7) Adams W, Ctercteko G, Bilous M. Effect of an omental wrap on the healing and vascularity of compromised intestinal anastomoses. Dis Colon Rectum 1992;35:731-738. 